\title{
Esclerite posterior: características clínicas, associação sistêmica, tratamento e evolução de 23 pacientes
}

\author{
Posterior scleritis: clinical features, systemic association, \\ treatment and evolution of 23 patients
}

\author{
Danuza de Oliveira Machado ${ }^{1}$ \\ André Luis Land Curi \\ Thaís Fontes Bessa ${ }^{3}$ \\ Wesley Ribeiro Campos ${ }^{4}$ \\ Fernando Oréfice ${ }^{5}$
}

Trabalho realizado no serviço de Uveítes do Hospital São Geraldo - Belo Horizonte (MG) - Brasil.

Pós-graduanda, nível Doutorado pela Universidade Federal de Minas Gerais - UFMG - Belo Horizonte (MG) - Brasil.

${ }^{2}$ Doutor em Oftalmologia pela UFMG - Belo Horizonte (MG) - Brasil

Assistente do Setor de Uveítes do Hospital São Geraldo - Hospital das Clínicas da UFMG - Belo Horizonte (MG) - Brasil.

${ }^{4}$ Chefe do Setor de Uveítes do Hospital São Geraldo Hospital das Clínicas da UFMG - Belo Horizonte (MG) Brasil.

${ }^{5}$ Professor Titular de Oftalmologia da UFMG - Belo Horizonte (MG) - Brasil.

Endereço para correspondência: Danuza de Oliveira Machado. Rua Professor Raimundo Cândido, 130/602 Belo Horizonte (MG) CEP 30320-650

E-mail: danuzadeoliveira@ig.com.br

Recebido para publicação em 27.09.2006

Última versão recebida em 30.06.2008

Aprovação em 21.05.2009

Nota Editorial: Depois de concluída a análise do artigo sob sigilo editorial e com a anuência da Dra. Áisa Haidar Lani sobre a divulgação de seu nome como revisora, agradecemos sua participação neste processo.

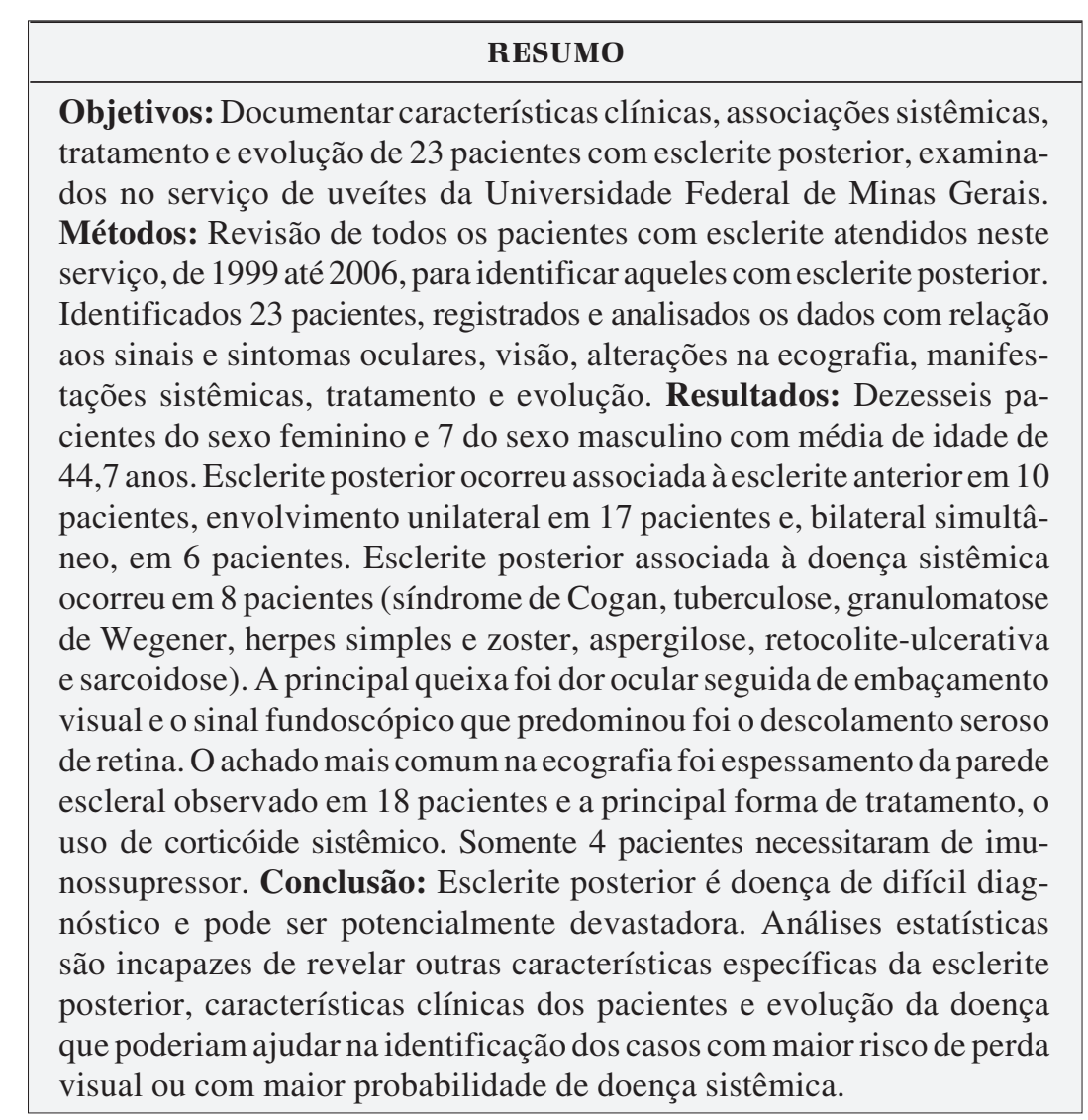

Descritores: Esclerite; Esclera/ultrassonografia; Descolamento retiniano; Dor; Ecografia; Prednisolona/uso terapêutico

\section{INTRODUÇÃO}

A esclerite posterior é uma forma de inflamação escleral considerada rara e significativamente pouco diagnosticada, mas em estudos recentemente publicados, o exame de ecografia B confirma que a esclerite posterior é quase tão comum quanto à esclerite anterior ${ }^{(1)}$. $\mathrm{O}$ quadro ocular pode ser bilateral em até $35 \%$ dos casos e a recorrência é frequente. Acomete preferencialmente mulheres de meia-idade ${ }^{(2-3)}$.

Por muitas vezes haver uma ausência de sinais fundoscópicos visíveis e pela proximidade da inflamação da mácula, retina e nervo óptico, o quadro 
escleral pode ser potencialmente devastador. O não reconhecimento e não tratamento de uma esclerite posterior pode comprometer a visão de maneira rápida e definitiva.

O quadro ocular pode ser idiopático ou estar associado a doenças sistêmicas em $45 \%$ dos $\operatorname{casos}^{(2)}$. Dentre as doenças mais frequentemente associadas à inflamação escleral podemos citar doenças infecciosas: herpes zoster oftálmico, herpes simples, sífilis, tuberculose (TBC); doenças reumáticas como artrite reumatóide e lúpus eritematoso sistêmico (LES); vasculites sistêmicas como granulomatose de Wegener, poliarterite nodosa, arterite de células gigantes e doença inflamatória intestinal como retocolite-ulcerativa e doença de $\mathrm{Crohn}^{(2-4)}$.

Seu diagnóstico é difícil e sempre deve ser levado em consideração no diagnóstico diferencial de condições tais como: glaucoma de ângulo fechado, dobras de coróide, edema de disco óptico, descolamento seroso de retina, descolamento de coróide, exsudação sub-retiniana, tumor intraocular e efusão uveal ${ }^{(4-5)}$.

A avaliação diagnóstica é importante em todos os casos de esclerite e inclui avaliação clínica geral, com interrogatório dos sistemas e investigação laboratorial. O encaminhamento para o reumatologista é aconselhável para cooperação no diagnóstico e tratamento.

Presença de dor ocular intensa, esclerite anterior, história de doença do tecido conjuntivo e vasculite sistêmica podem ajudar o oftalmologista no diagnóstico correto. A ecografia B é indispensável para o seu diagnóstico, principalmente quando não há sinais fundoscópicos evidentes ${ }^{(6-7)}$.

Pretendeu-se neste estudo, descrever as características clínicas, associações sistêmicas, tratamento e evolução de 23 pacientes com esclerite posterior, atendidos no serviço de uveítes da Universidade Federal de Minas Gerais.

\section{MÉTODOS}

Uma revisão retrospectiva dos prontuários médicos de todos os pacientes com esclerite, atendidos nos serviço de uveítes da Universidade Federal de Minas Gerais entre os anos de 1999 e 2006, foi realizada para identificar aqueles pacientes com diagnóstico de esclerite posterior. Foram identificados 23 pacientes ( 29 olhos) e todos, foram incluídos no trabalho. O diagnóstico de esclerite posterior foi utilizado como critério de inclusão dos pacientes neste trabalho. Não houve critérios de exclusão. Dezenove pacientes ainda estavam em acompanhamento no serviço e desses, 11 ainda estavam em tratamento.

Detalhes com relação ao início da doença, queixas dos pacientes, sinais e sintomas clínicos e oculares, alterações na ecografia $\mathrm{B}$, associação com doença sistêmica, tratamento e evolução do quadro foram registrados e analisados pelo programa estatístico SPSS (análise descritiva - frequência). Os dados dos pacientes foram coletados em uma única ocasião (corte transversal) e analisados ao longo de três meses (de junho de 2006 a setembro de 2006). A data final para a inclusão de novos pacientes foi 30 de setembro de 2006.
O trabalho foi aprovado pelo Departamento de Oftalmologia-Otorrinolaringologia e Fonoaudiologia e também foi aprovado pelo Comitê de Ética em Pesquisa da UFMG (COEP) sob o parecer número ETIC 243/07.

\section{RESULTADOS}

Dos 23 pacientes que participaram deste estudo, 69,5\% eram do sexo feminino e $30,5 \%$ do sexo masculino. A média de idade foi de 44,7 anos variando de 12 a 65 anos.

A esclerite posterior ocorreu sem associação com esclerite anterior, no momento da apresentação, em 13 pacientes $(56,5 \%)$ e associada à esclerite anterior, em $10(43,5 \%)(30,4 \%$ com esclerite anterior difusa, $8,7 \%$ com esclerite anterior nodular, $4,3 \% \mathrm{com}$ esclerite anterior necrosante) (Gráfico 1). Nenhum dos 13 pacientes que apresentou esclerite posterior isolada evoluiu, durante o período de acompanhamento, com esclerite anterior.

O quadro escleral foi recorrente em 6 pacientes $(26,1 \%)$. Envolvimento unilateral, no momento do diagnóstico, ocorreu em 17 pacientes $(73,9 \%)$ (17 olhos) e bilateral simultâneo, em $6(26,1 \%)$ (12 olhos).

Dos 23 pacientes que foram analisados, 15 (65,2\%) apresentou esclerite posterior idiopática e 8 pacientes $(34,8 \%)$, associação com doença sistêmica e infecção. As doenças associadas estão detalhadas na tabela 1 . O diagnóstico de doença sistêmica foi posterior ao quadro de esclerite em $21,7 \%$ dos pacientes.

Dor ocular, dor à movimentação ocular, cefaléia, embaçamento visual e sinais e sintomas relacionados à esclerite anterior são frequentemente observados na esclerite posterior. Com relação aos 23 pacientes deste trabalho, $22(95,6 \%)$ relataram dor ocular intensa, $16(69,5 \%)$ embaçamento visual e 15 $(65,2 \%)$, apresentaram queixas associadas à esclerite anterior como hiperemia, fotofobia e lacrimejamento.

Os sinais clínicos fundoscópicos da esclerite posterior foram variados. $\mathrm{O}$ sinal mais encontrado foi o descolamento seroso de retina que ocorreu em 15 pacientes $(65,2 \%)$, seguido de edema de disco óptico em 6 pacientes $(26,1 \%)$, infiltrados sub-retinianos em $6(21,1 \%)$ pacientes e 1 caso $(4,34 \%)$ com descolamento seroso de retina associado a um descolamento gigante de coróide e síndrome de efusão uveal.

No exame de ecografia a alteração mais observada foi o espessamento esclerocoroidiano (espessamento da parede escleral posterior), ocorrendo em 18 pacientes $(78,2 \%)$. O clássico, mas não patognomônico, sinal do T (fluído no interior da cápsula de Tenon e na bainha do nervo óptico determinando uma letra $\mathrm{T}$ escura na ecografia. A barra horizontal do $\mathrm{T}$ é formada pela ecoluscência do fluído na cápsula de tenon e a barra vertical pela ecoluscência do disco óptico de aspecto dilatado) foi detectado em apenas 7 casos (30,4\%) (Figura 1).

A perda visual é a complicação mais temida na esclerite posterior. Baixa da acuidade visual de duas ou mais linhas ocorreu em 19 pacientes $(82,6 \%)$, mas com o tratamento, a melhora da visão foi extremamente satisfatória em 14 (60,8\%) 


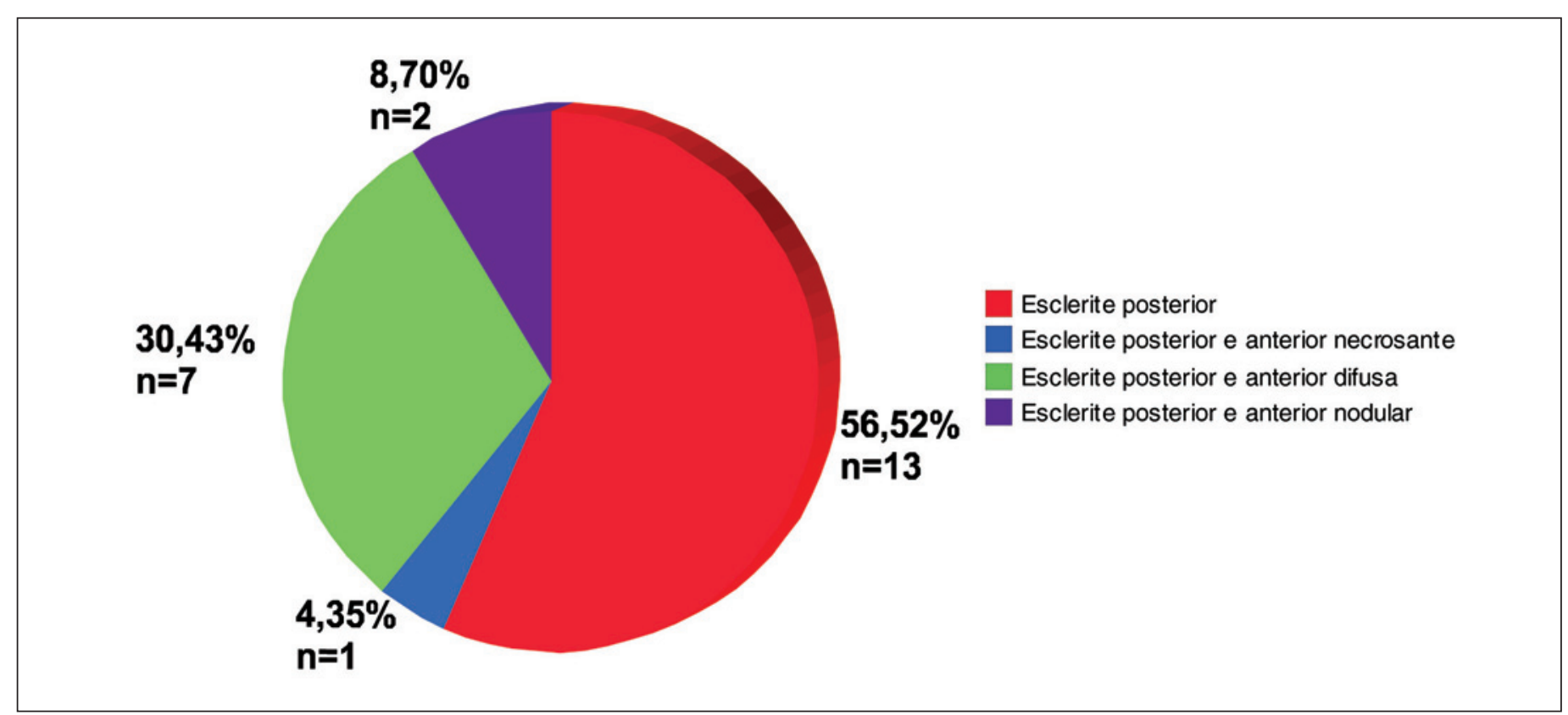

Gráfico 1 - Classificação das esclerites

\begin{tabular}{|lc|}
\hline Tabela 1. Doenças sistêmicas associadas à esclerite posterior \\
Doença sistêmica & Número de pacientes \\
Sarcoidose & 1 \\
Síndrome de Cogan & 1 \\
Herpes vírus & 2 \\
Retocolite-ulcerativa & 1 \\
Granulomatose de Wegener & 1 \\
Tuberculose & 1 \\
Aspergilus & 1 \\
\hline
\end{tabular}

sendo que, em alguns, houve recuperação total da visão perdida. A paciente com descolamento gigante da coróide e síndrome de efusão uveal evoluiu mal, sem melhora da acuidade visual e a paciente cuja biópsia escleral mostrou a presença de fungo acabou evoluindo para a enucleação do olho afetado devido à piora do quadro ocular e perda visual. Uma paciente não teve mais nenhum registro no prontuário médico após suas primeiras consultas, não realizou tratamento neste serviço e, portanto não conhecemos a sua evolução e acuidade visual final. Duas pacientes abandonaram o serviço no meio do tratamento.

Dos 10 pacientes que apresentaram esclerite anterior associada ao quadro posterior, três evoluíram com afilamento escleral importante no setor de comprometimento da esclera anterior (um paciente apresentava esclerite anterior necrosante associada à sarcoidose, outro paciente, apresentava esclerite anterior difusa e posterior diagnóstico de granulomatose de Wegener e a terceira paciente apresentou episódio de esclerite anterior nodular com grande nódulo escleral no olho afetado e achado ocasional de esclerite posterior na ecogra-

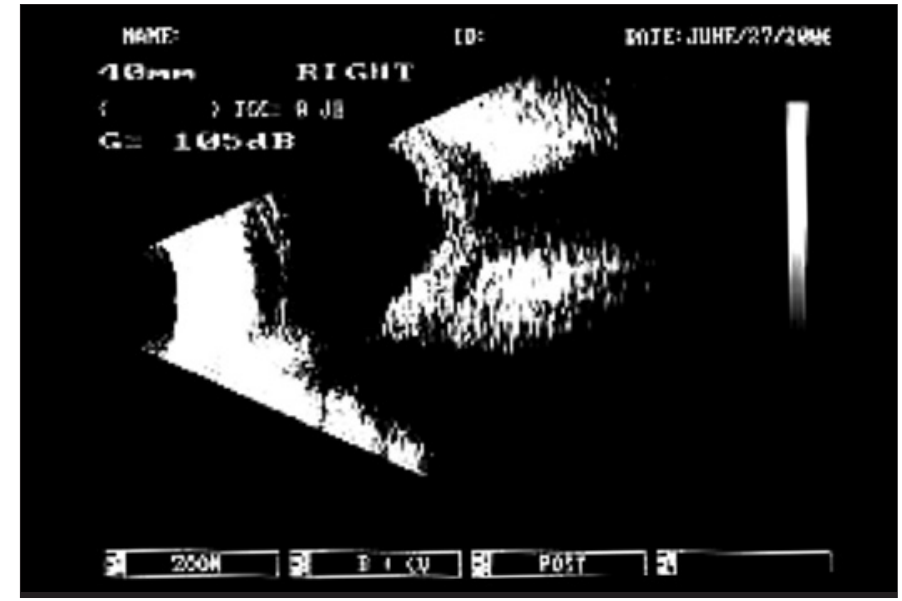

Figura 1 - Sinal do T

fia). Um paciente evoluiu com aumento da transparência escleral na região superior, bilateralmente (apresentava esclerite anterior difusa bilateral), dois pacientes evoluíram com catarata e um com glaucoma. Em resumo, na tabela 2, estão algumas características dos pacientes com esclerite posterior.

A principal forma de tratamento instituída foi o uso de corticosteróide oral (1 mg/kg/dia) em 16 pacientes $(69,5 \%)$, sendo que em um deles foi associado o aciclovir oral devido ao diagnóstico de herpes e em outro, um antifúngico. Quatro pacientes $(17,4 \%)$ iniciaram o tratamento com DAINE oral (naproxeno), mas como não houve melhora do quadro ocular em três, o corticosteróide oral foi introduzido. Já em outro paciente, segundo os registros no prontuário, a única forma de tratamento realizada foi o uso de corticosteróide tópico. 


\begin{tabular}{|lcc|}
\hline \multicolumn{2}{|l}{$\begin{array}{l}\text { Tabela 2. Características dos pacientes com esclerite posterior } \\
\text { Características }\end{array}$} & $\begin{array}{c}\text { Porcentagem } \\
\text { 95\% Intervalo } \\
\text { de confiança }\end{array}$ \\
Média de idade (anos) & $44,7 \%$ & $39,4-50,0$ \\
Sexo feminino & $69,5 \%$ & $47,1-86,8$ \\
Unilateral & $73,9 \%$ & $51,6-89,8$ \\
Esclerite anterior associada & $43,5 \%$ & $23,2-65,5$ \\
Dor ocular & $95,6 \%$ & $78,1-99,9$ \\
Descolamento seroso de retina & $69,6 \%$ & $47,1-86,8$ \\
Esclerite posterior idiopática & $65,2 \%$ & $47,2-83,6$ \\
Doença sistêmica associada & $34,8 \%$ & $16,4-57,3$ \\
\hline
\end{tabular}

Foi necessária a pulsoterapia venosa com metilprednisolona, como tratamento inicial $(1 \mathrm{~g} /$ dia por três dias consecutivos), em um paciente que além da esclerite apresentava quadro de pseudotumor inflamatório. O paciente com granulomatose de Wegener, antes de receber o seu diagnóstico, iniciou tratamento com corticosteróide oral ( $1 \mathrm{mg} / \mathrm{kg} / \mathrm{dia})$, seguido de pulsoterapia venosa com metilprednisolona $(1 \mathrm{~g} /$ dia por três dias consecutivos) e ciclosporina oral (4 m/ $\mathrm{kg} / \mathrm{dia}$ ) (apresentava quadro bilateral e grave de esclerite anterior e posterior). Houve boa resposta com melhora do quadro ocular, mas após o diagnóstico, o seu tratamento foi modificado pela reumatologia, para pulsoterapia venosa com ciclofosfamida devido ao aparecimento de quadro pulmonar.

Três pacientes apresentaram quadros recorrentes com a diminuição da dose do corticosteróide oral e por isso, foi necessária a introdução de droga imunossupressora para controle da esclerite. Em um paciente foi iniciada a ciclosporina ( $4 \mathrm{mg} / \mathrm{kg} / \mathrm{dia})$ e nos outros dois, a azatioprina $(2,5 \mathrm{mg} / \mathrm{kg} / \mathrm{dia})$ com excelente resposta em todos os casos (Tabela 3 ).

\section{DISCUSSÃO}

A esclerite posterior é uma doença rara, grave e de difícil diagnóstico. O conhecimento sobre suas características clínicas e formas de apresentação são de extrema importância na prática oftalmológica, pois o diagnóstico correto e tratamento precoce dessa doença podem evitar a perda visual.

No presente trabalho, a idade média dos pacientes com esclerite posterior foi de 44,7 anos. A esclerite posterior se iniciou antes dos 40 anos de idade em $43,5 \%$ dos casos. Tais resultados estão de acordo com diversos trabalhos nos quais, pacientes com esclerite posterior, são predominantemente de meia idade e a idade média para o início do quadro é de 49 $\operatorname{anos}^{(2,4,8)}$. Em somente $30 \%$ dos casos, ela pode ocorrer antes dos 40 anos de idade ${ }^{(9)}$.

A frequência de esclerite posterior em crianças e adultos jovens é baixa, mas existe relato do encontro de esclerite posterior simulando um tumor intraocular em uma criança de apenas cinco anos de idade ${ }^{(10-11)}$. O paciente mais novo que tivemos foi um menino de 12 anos de idade com um quadro unilateral de esclerocerato-uveíte herpética e edema de disco óptico.

\begin{tabular}{|lcc|}
\hline \multicolumn{3}{|c|}{ Tabela 3. Tratamento das esclerites } \\
Tratamento & Frequência & Porcentagem \\
Corticóide oral & 10 & $43,5 \%$ \\
Corticóide oral e aciclovir & 1 & $4,3 \%$ \\
Corticóide oral e antifúngico & 1 & $4,3 \%$ \\
Corticóide oral seguido de azatioprina & 2 & $8,7 \%$ \\
Corticóide oral seguido de ciclosporina & 1 & $4,3 \%$ \\
Corticóide oral seguido de & 1 & $4,3 \%$ \\
pulsoterapia metilprednisolona e & & \\
ciclosporina e após diagnóstico & & \\
de Wegener, pulso de ciclofosfamida & & \\
DAINE oral & 4 & $17,4 \%$ \\
Corticóide tópico & 1 & $4,3 \%$ \\
Nenhum tratamento & 1 & $4,3 \%$ \\
Pulso de Metilprednisolona & 1 & $4,3 \%$ \\
Total & 23 & $100,0 \%$ \\
*DAINE= droga anti-inflamatória não-esteróide & \\
\hline
\end{tabular}

O trabalho mostrou que a esclerite posterior foi 2,5 vezes mais comum no sexo feminino que no sexo masculino, o que também está de acordo com a literatura que cita $65 \%$ dos pacientes com esclerite posterior, sendo mulheres ${ }^{(2,9,12)}$. Interessantemente, em uma pequena série de pacientes (47) analisados em um artigo, houve preponderância de esclerite posterior no sexo masculino e em jovens (média de 19 anos) ${ }^{(13)}$.

Segundo alguns autores, pacientes com mais de 50 anos de idade e esclerite apresentam maior risco de associação com doença sistêmica e perda visual ${ }^{(14-16)}$. Também mostram nos trabalhos citados, que não há relação entre o fato de a doença escleral ser unilateral, bilateral e recidivante com a presença de doença sistêmica ou perda visual. A maioria dos pacientes do presente trabalho $(73,9 \%)$ evoluiu com doença unilateral no momento da apresentação. Em 34,8\% dos casos, os pacientes apresentaram associação com doença sistêmica concordando com a literatura que afirma um risco entre 30 a $45 \%$ de associação entre esclerite posterior e doença sistêmica ${ }^{(16)}$. Em 62,5\%, os pacientes tinham entre 45 a 65 anos de idade. Cinco dos pacientes com doença sistêmica apresentavam doença unilateral e três, doença bilateral no momento da apresentação. Somente um deles, até o momento, apresentou recidiva da inflamação escleral.

Dentre as doenças mais frequentemente associadas à inflamação escleral, podemos citar doenças infecciosas tais como, herpes zoster oftálmico, herpes simples, sífilis, $\mathrm{TBC}^{(17)}$; doenças reumáticas como artrite reumatóide, LES $^{(18)}$; vasculites sistêmicas como granulomatose de Wegener, poliarterite nodosa, arterite de células gigantes e doença inflamatória intestinal como retocolite-ulcerativa e doença de $\mathrm{Crohn}^{(19)}$. Neste trabalho, houve predomínio da esclerite posterior idiopática (56,3\% dos casos). As doenças sistêmicas diagnosticadas foram predominantemente vasculites sistêmicas ou de causa infecciosa (sarcoidose, síndrome de Cogan, herpes simples e zoster, retocolite-ulcerativa, tuberculose, granulomatose de Wegener e aspergilose). A esclerite associada à 
infecção pelo vírus herpes ocorreu em dois pacientes confirmando a infecção herpética como principal causa infecciosa de esclerite ${ }^{(17)}$.

Quarenta e três e meio por cento $(43,5 \%)$ dos pacientes avaliados apresentavam esclerite posterior associada à esclerite anterior no momento do diagnóstico, incidência considerada mais alta do que a previamente documentada ${ }^{(9)}$. Pacientes que desenvolvem esclerite anterior associada à esclerite posterior têm um risco aumentado para doença sistêmica ${ }^{(4)}$. No presente trabalho, $60,0 \%$ dos pacientes com associação entre esclerite anterior e posterior, apresentavam doença sistêmica.

Todos os pacientes desta série eram sintomáticos no momento do diagnóstico sendo a dor ocular $(95,7 \%)$ e o embaçamento visual $(69,6 \%)$ as queixas mais comuns. A dor ocular é considerada o principal sintoma de uma esclerite e além de intensa, pode envolver não só olho e a órbita, mas irradiar-se para a cabeça, ouvidos e mandíbulas. Ela geralmente piora com a movimentação ocular e pode despertar o paciente do sono ${ }^{(9)}$. Alguns pacientes com esclerite posterior podem não apresentar dor ${ }^{(20)}$.

Dentre os sinais clínicos destacaram-se o descolamento seroso de retina $(65,3 \%)$, edema de disco óptico $(26,1 \%)$ e, infiltrados sub-retinianos $(26,1 \%)$. Em uma paciente $(4,3 \%)$ havia um descolamento seroso de retina associado a um descolamento gigante de coróide e síndrome de efusão uveal, em outra paciente não havia manifestação fundoscópica da esclerite posterior que foi confirmada apenas pela ecografia. Os sinais e sintomas de uma esclerite posterior dependem da localização e gravidade da inflamação, extensão do quadro e relação com as estruturas oculares que estão ao seu redor, tais como músculos extraoculares, coróide, retina e disco óptico ${ }^{(4)}$. Portanto, inflamações periféricas e comprometendo de maneira profunda a esclera, podem levar ao aparecimento de efusão uveal e glaucoma ${ }^{(12)}$. Inflamação escleral nodular localizada pode produzir massa sub-retiniana com descolamento seroso de retina, já a inflamação próxima ao disco óptico e mácula pode levar a edema de disco óptico, descolamento seroso envolvendo a macula e dobras na coróide e retina ${ }^{(4)}$.

Apenas quatro dos pacientes avaliados apresentaram uveíte anterior leve (até $2+$ de células na câmara anterior) associada ao quadro escleral. Nenhum dos pacientes apresentou uveíte anterior grave como parte do quadro ocular. Segundo alguns autores, uveíte anterior grave não seria característica clínica de nenhuma das esclerites e se presente, o diagnóstico deveria ser reconsiderado ${ }^{(3-4,21)}$.

A ecografia B é extremamente importante no diagnóstico da esclerite posterior. Se realizada por uma pessoa experiente, pode inclusive classificar a esclerite posterior em difusa, nodular ou necrosante ${ }^{(1)}$. $\mathrm{O}$ espessamento da parede escleral posterior (maior que $2 \mathrm{~mm}$ ) é o sinal ecográfico mais comum na esclerite posterior ${ }^{(9,22)}$. Esse sinal ocorreu em $78,3 \%$ dos pacientes avaliados no presente trabalho. O clássico sinal do $\mathrm{T}$ foi observado em apenas $30,4 \%$ dos casos. Na paciente com doença escleral associada à presença de fungo (Aspergillus) $\mathrm{e}$ no paciente com granulomatose de Wegener, a ecografia B mostrou a presença de nódulo escleral posterior, classificando a esclerite posterior como nodular.

O tratamento da esclerite posterior pode utilizar drogas anti-inflamatórias não-esteróides (DAINE) orais, corticosteróide oral e nos casos graves e resistentes ao tratamento convencional, imunossupressores. A principal forma de tratamento é o corticosteróide oral com bom controle do quadro ocu$\operatorname{lar}^{(2,8-9,20,23)}$. No presente estudo, 69,6\% dos pacientes foram submetidos a tratamento inicial com corticosteróide oral e $17,4 \%$ com DAINE oral. Em apenas $4(17,4 \%)$ pacientes foi necessária a introdução de imunossupressor oral (azatioprina ou ciclosporina) para o controle do quadro, sendo que 3 deles apresentavam doença sistêmica associada (retocolite-ulcerativa, sarcoidose, granulomatose de Wegener). Pacientes com baixa visual grave, evidência de envolvimento do nervo óptico e associação com doença sistêmica geralmente necessitam de terapia anti-inflamatória mais agressiva, incluindo o uso de imunossupressor ${ }^{(4,24)}$.

O principal risco de uma esclerite posterior é a perda definitiva da visão. Felizmente houve melhora da acuidade visual (em duas ou mais linhas na escala de AV de Snellen) na quase totalidade dos nossos pacientes, mas vários outros estudos mostram que o risco de perda visual é alto ${ }^{(8)}$. A paciente com esclerite fúngica, evoluiu de maneira dramática para a enucleação do olho afetado.

\section{CONCLUSÃO}

Análises estatísticas foram incapazes de revelar outras características específicas da apresentação da esclerite posterior, características clínicas dos pacientes e evolução da doença que poderiam ajudar na identificação dos casos com maior risco de perda visual ou com maior probabilidade de associação com doença sistêmica. Todos os pacientes devem ser considerados de risco, portanto, a propedêutica e terapêutica devem ser instituídas para todos, tão logo seja feito o diagnóstico. Como a esclerite posterior é doença de difícil diagnóstico e com grande número de diagnósticos diferenciais, ela deve sempre ser lembrada como causa de inflamação ocular. Isso é fundamental para a detecção desta doença potencialmente destrutiva.

\section{ABSTRACT}

Purpose: To document the clinical features, systemic association, treatment and evolution of 23 patients with posterior scleritis evaluated in the Uveitis service of the Federal University of Minas Gerais. Methods: 23 patients were identified with the diagnosis of posterior scleritis. Signals and symptoms, visual acuity, B-mode ultrasonography signals, systemic associations, treatment and evolution were described and analyzed. Results: Sixteen patients were female and seven were male with mean age of 44,7 years. Posterior scleritis occurred in association with anterior scleritis in 10 patients, unilateral 
involvement in 17 patients and simultaneous bilateral involvement in 6 patients. Posterior scleritis in association with systemic disease occurred in 8 patients (Cogan's syndrome, TBC, Wegener, Herpes simplex and Zoster, Apergilosis, inflamatory bowel disease and Sarcoidosis). The main symptoms were ocular pain and decrease of visual acuity and the main signal was retinal serous detachment. Increase of thickness choroidal tissue was the main signal in B-mode ultrasonography in 18 patients and the principal kind of treatment was the use of systemic corticosteroids. Only 4 patients required systemic immunosuppressive drugs. Conclusions: Posterior scleritis still represents a diagnostic challenge and is often associated with life threatening systemic disease and vision threatening ocular complications. Knowledge of posterior scleritis may aid in determining timely and accurate diagnosis and treatment of both ocular and any systemic conditions associated, thus decreasing morbidity and mortality. Elevated suspicion rate is always required to detect this condition.

Keywords: Scleritis; Sclera/ultrasonography; Retinal detachment; Pain; Prednisolone/therapeutic use

\section{REFERÊNCIAS}

1. Watson PG. The management of inflammatory scleral disease. Contemp Ophthalmol. 2003;2:1-7.

2. Watson PG, Hazleman BL, Pavésio CE, Green WR. Clinical presentation of scleritis and episcleritis. In: Watson PG, Hazleman BL, Pavésio CE, Green WR. The sclera and systemic disorders. $2^{\text {nd }}$ ed. Philadelphia: WB Saunders; 2004.

3. Oréfice F, Pavésio CE. Esclerites. In: Oréfice F, editor. Uveíte clínica e cirúrgica. $2^{\mathrm{a}}$ ed. Rio de Janeiro: Cultura Médica; 2005. p.977-86.

4. McCluskey PJ, Watson PG, Lightman S, Haybittle J, Restori M, Branley M. Posterior scleritis: clinical features, systemic associations, and outcome in a large series of patients. Ophthalmology. 1999;106(12):2380-6.
5. Sainz de la Maza M, Jabbur NS, Foster CS. Severity of scleritis and episcleritis. Ophthalmology. 1994;101(2):389-96.

6. Biswas J, Mittal S, Ganesh SK, Shetty NS, Gopal L. Posterior scleritis: clinical profile and imaging characteristics. Indian J Ophthalmol. 1998;46(4): 195-202.

7. Perri P, Mazzeo V, De Palma P, Pastena B, Police G, Ravalli L, Rossi A. Posterior scleritis: ultrasound findings in two cases. Ophthalmologica. 1998; 212(Suppl 1):110-2.

8. Jabs DA, Mudun A, Dunn JP, Marsh MJ. Episcleritis and scleritis: clinical features and treatment results. Am J Ophthalmol. 2000;130(4):469-76.

9. Okhravi N, Odufuwa B, McCluskey P, Lightman S. Scleritis. Surv Ophthalmol. 2005;50(4):351-63.

10. Jensen JE, Fledelius HC, Prause JU, Scherfig E. An unusual ophthalmic tumour in a 5-year-old boy. Acta Ophthalmol Suppl. 1992(204):110-2.

11. Wald KJ, Spaide R, Patalano VJ, Sugin S, Yannuzzi LA. Posterior scleritis in children. Am J Ophthalmol. 1992;113(3):281-6.

12. Benson WE. Posterior scleritis. Surv Ophthalmol. 1988;32(5):297-316.

13. Calthorpe CM, Watson PG, McCartney AC. Posterior scleritis: a clinical and histological survey. Eye. 1988;2(Pt 3):267-77.

14. Akpek EK, Thorne JE, Qazi FA, Do DV, Jabs DA. Evaluation of patients with scleritis for systemic disease. Ophthalmology. 2004;111(3):501-6. Comment in: Ophthalmology. 2007;114(6):1232.

15. Akpek EK, Uy HS, Christen W, Gurdal C, Foster CS. Severity of episcleritis and systemic disease association. Ophthalmology. 1999;106(4):729-31.

16. Pavésio CE, Meier FM. Systemic disorders associated with episcleritis and scleritis. Curr Opin Ophthalmol. 2001;12(6):471-8.

17. Sainz de la Maza M, Hemady RK, Foster CS. Infectious scleritis: report of four cases. Doc Ophthalmol. 1993;83(1):33-41.

18. Sainz de la Maza M, Foster CS, Jabbur NS. Scleritis associated with rheumatoid arthritis and with other systemic immune-mediated diseases. Ophthalmology. 1994;101(7):1281-6; discussion 1287-8.

19. Sainz de la Maza M, Foster CS, Jabbur NS. Scleritis associated with systemic vasculitic diseases. Ophthalmology 1995;102(4):687-92.

20. Sainz de la Maza M. Episcleritis and sleritis. Contemp Ophthalmol. 2006; $5(9): 1-8$.

21. Sainz de la Maza M, Foster CS, Jabbur NS. Scleritis-associated uveitis. Ophthalmology. 1997;104(1):58-63.

22. Baráková D, Bujalková D, Redinová M. [Echographic findings in posterior scleritis]. Cesk Slov Oftalmol 2003;59(2):86-90. Czech.

23. Olivalves SM, Petrille AM, Martins MC, Kim MK, Abreu, MT, Hilário, ME, et al. Uveíte associada a artrite reumatóide juvenil. Arq Bras Oftalmol. 1993; 56(5):263-5.

24. Orefice F, Carvalho MAR. Uveítes e artrites. Arq Bras Oftalmol. 1995;48(4): 123-9. 